\title{
Eficacia del tratamiento quirúrgico mediante neurotización radial en pacientes con lesión traumática aislada de nervio axilar
}

\author{
Polanco-Armenta AG, *** Solano-Pérez RJ, ${ }^{*}$ Sánchez-Prado MG, ${ }^{*}$ Pérez-Atanasio JM,* Torres-González R* \\ Unidad Médica de Alta Especialidad «Dr. Victorio de la Fuente Narváez» Hospital de Traumatología, Ortopedia y Rehabilitación, \\ Instituto Mexicano del Seguro Social. Ciudad de México
}

RESUMEN. Objetivo: Determinar la eficacia del tratamiento quirúrgico mediante neurotización radial en pacientes con diagnóstico de lesión traumática aislada de nervio axilar. Material y métodos: Se presenta una serie de siete casos, seis hombres y una mujer, con promedio de edad de 51.6 años, con diagnóstico de lesión traumática aislada de nervio axilar tratados quirúrgicamente entre Enero 2013 y Diciembre 2016. Todos los pacientes fueron tratados mediante transferencia nerviosa de rama motora radial de la cabeza medial del tríceps, entre el sexto y decimoséptimo mes después del traumatismo, con un seguimiento mínimo de 12 meses. Se evaluó la eficacia del tratamiento mediante la Medical Research Council (MRC) y el cuestionario DASH. Resultados: En el análisis bivariado encontramos diferencias estadísticamente significativas con respecto al grado de mejoría de fuerza de rotación externa y de abducción medido por MRC preoperatoria y postoperatoria $(p<0.05)$. Los resultados también demostraron una significancia estadística en el rango de movimiento de abducción postoperatorio de hombro $(p=0.01)$. El cuestionario DASH demostró un porcentaje promedio de discapacidad de $20.29 \%$ después de 12 meses de seguimiento. Conclusión: La neurotización radial en pacientes con diagnóstico de lesión traumática aislada de nervio axilar proporciona un excelente grado de mejoría de fuerza de rotación externa y
ABSTRACT. Purpose: To determine the efficacy of surgical treatment by radial nerve transfer in patients with a diagnosis of isolated axillary nerve traumatic injury. Material and methods: We present a series of seven cases, six men and one woman, with a mean age of 51.6 years, with a diagnosis of isolated traumatic axillary nerve injury between January 2013 and December 2016. All patients were treated by radial motor branch nerve transfer of the medial triceps head between the sixth and seventeenth months after trauma, with a minimum follow-up of 12 months. The efficacy of the treatment was evaluated by the Medical Research Council (MRC) and the DASH questionnaire. Results: In the bivariate analysis we found statistically significant differences regarding the degree of external rotation and abduction strength measured by preoperative and postoperative MRC $(p<0.05)$. The results also demonstrated a statistical significance in the range of postoperative shoulder abduction movement $(p=0.01)$. The DASH questionnaire showed an average percentage of disability of $20.29 \%$ after 12 months of follow-up. Conclusion: Radial nerve transfer in patients diagnosed with axillary nerve traumatic injury provides an excellent degree of improvement in external rotation and abduction strength, in addition to significantly improving the shoulder abduction range, it was

Nivel de evidencia: IV

\footnotetext{
* Clínica de Cirugía de Plexo Braquial y Nervio Periférico, Unidad Médica de Alta Especialidad «Dr. Victorio de la Fuente Narváez». Hospital de Traumatología, Ortopedia y Rehabilitación. Instituto Mexicano del Seguro Social, Ciudad de México.

** Facultad de Medicina, Universidad Nacional Autónoma de México. Ciudad de México.

Dirección para correspondencia:

Alan Giovanni Polanco-Armenta

Av. Colector Núm. 15 esquina con Av. Instituto Politécnico Nacional, Col. Magdalena de las Salinas, CP 07760,

Delegación Gustavo A. Madero, Ciudad de México.

Teléfono: 5519527551

E-mail: alan.giovanni.polar@gmail.com
}

Este artículo puede ser consultado en versión completa en http://www.medigraphic.com/actaortopedica 
abducción, además de mejorar significativamente el rango de abducción del hombro; también se demostró, mediante cuestionario DASH, que el porcentaje de discapacidad es bajo.

Palabras clave: Lesión de nervio axilar, transferencia nerviosa, nervio radial, parálisis, abducción, hombro.

\section{Introducción}

La lesión aislada del nervio axilar es una entidad poco frecuente, debido a que la mayoría de estas lesiones se presentan asociadas a una afectación más extensa del plexo braquial. ${ }^{1} \mathrm{La}$ incidencia de las lesiones de nervio axilar infraclaviculares se estima en $0.3-6 \%$ del total de lesiones de plexo braquial. ${ }^{1,2}$ Las lesiones de nervio axilar o plexo braquial pueden resultar de traumatismos penetrantes, lesiones por tracción, luxación glenohumeral y como complicaciones de cirugía de hombro. ${ }^{1,3}$ Como mecanismo de lesión más frecuente se ha descrito el traumatismo cerrado sobre región escapulohumeral, asociándose habitualmente a lesiones osteoarticulares y/o de los músculos del manguito rotador. ${ }^{4,5}$

La consiguiente falta de abducción del hombro secundaria a una lesión nerviosa puede ocasionar un déficit funcional severo para los pacientes, lo que provoca que las actividades de la vida cotidiana sean difíciles o imposibles. ${ }^{6,7} \mathrm{La}$ mayoría de las lesiones del nervio axilar evolucionan hacia la recuperación espontánea en los primeros seis meses, $, 1,3,5,8,9$ calculándose que en menos de $20 \%$ de los casos la parálisis persiste clínicamente sin evidencia de recuperación neurofisiológica. ${ }^{2,5,10}$

La técnica quirúrgica más utilizada en los trabajos publicados hasta la fecha es la reparación con injerto, ${ }^{9,11,12}$ seguida de la neurólisis y de la sutura directa. Debido a la tasa enormemente alta de buenos resultados que brindan las transferencias distales se han transformado en el procedimiento de elección..$^{4,9,13}$ La transferencia nerviosa, también llamada neurotización o cruce de nervios, consiste en seccionar un nervio o rama normal y conectar su muñón proximal al muñón distal de un nervio lesionado. ${ }^{8}$ Esto implica el sacrificio de un nervio sano cuya función debe ser compensado por el resto de músculos inervados. ${ }^{13}$ Esta compensación funcional puede ser promovida por agonista simple, por hipertrofia muscular o, en caso de denervación parcial, mediante el desarrollo de unidades motrices después del brote axonal terminal en el nivel del músculo. ${ }^{14}$ Las transferencias nerviosas se utilizan cuando un muñón del nervio proximal no está disponible para repararlo. ${ }^{6,13}$

La transferencia de la rama de la cabeza larga del tríceps al nervio axilar primero fue utilizada con éxito por Colemann en 1946, según lo notificado por Carayon y Bou- also demonstrated by DASH questionnaire that the percentage disability is low.

Key words: Isolated axillary nerve injury, nerve transfer, radial nerve, paralysis, abduction, shoulder.

rrel. ${ }^{15}$ Lurje informó un caso único en el que dos tríceps sin nombre de las ramas se diseccionaron utilizando un enfoque deltopectoral y se conectaron al nervio axilar. ${ }^{16}$ Nath y Mackinnon, utilizando un enfoque axilar similar y después de disección intraneural del nervio radial, transfirieron los fascículos tríceps al nervio axilar. ${ }^{7,9}$ Más recientemente, Leechavengvongs y colaboradores, ${ }^{17}$ reportaron de forma independiente la transferencia de la rama motora a la cabeza larga del tríceps usando un abordaje posterior después del traumatismo del plexo braquial; también demostraron resultados similares con la transferencia de la rama a la cabeza lateral del tríceps. ${ }^{18}$

El cuestionario discapacidad de brazo, hombro y mano (DASH) es una de las escalas más utilizadas para determinar la funcionalidad y discapacidad de miembro superior. Se ha empleado en población general, demostrando una alta consistencia interna y fiabilidad test-retest, por lo que se considera un instrumento válido y fiable para evaluar discapacidad, ${ }^{19}$ donde se incluye la evaluación de diferentes articulaciones de extremidad superior con aplicación en pacientes postoperados de neurotización radial en lesión de nervio axilar.

El objetivo de este estudio fue analizar los resultados de una serie de siete casos de lesiones puras de nervio axilar producidas como consecuencia de una luxación traumática de hombro sin lesión de plexo asociada, en las cuales se realizó una reparación quirúrgica mediante transferencia de ramo de la porción larga del tríceps al nervio axilar. Se evaluó la eficacia del tratamiento mediante la Medical Research Council ${ }^{20}$ (MRC) y el cuestionario DASH.

\section{Material y métodos}

Se realiza una revisión retrospectiva de una serie de siete casos de los pacientes con diagnóstico de lesión traumática aislada de nervio axilar intervenidos quirúrgicamente mediante transferencia nerviosa de la rama radial de la porción larga del tríceps al nervio axilar en el período del 1 de Enero de 2013 a 31 de Diciembre de 2016. Se descartaron pacientes que presentaban lesiones de nervio axilar asociadas con otra lesión nerviosa o de plexo braquial, así como los que no tenían un seguimiento mínimo de un año. Siete de los pacientes cumplieron con los criterios de selección: seis hombres y una mujer, con un promedio de edad de 51 
años. La etiología de la lesión de nervio axilar fue una luxación traumática de la articulación escapulohumeral en todos los casos. La transferencia nerviosa de la porción larga del tríceps al nervio axilar se llevó a cabo entre el mes seis y 17 después del traumatismo por ausencia de recuperación clínico-neurofisiológica espontánea.

Se revisaron los expedientes médicos de los pacientes para obtener: datos demográficos incluyendo edad, sexo, fecha de la lesión, mecanismo de lesión y tiempo para la cirugía; los datos clínicos, incluidas las evaluaciones de la fuerza y de la abducción preoperatoria; datos quirúrgicos incluyendo procedimientos realizados y complicaciones; y los datos de seguimiento, incluidas la duración, medición de fuerza de abducción y abducción activa del hombro. Los cirujanos responsables realizaron todas las evaluaciones. La fuerza fue evaluada de acuerdo con la British Medical Research Council (MRC). La disminución de la movilidad de hombros fue medido por el cirujano tratante con un goniómetro y se definió como el ángulo entre el eje del brazo y la columna toracolumbar.

La técnica utilizada fue la siguiente: el paciente en decúbito ventral, colocándose un realce en la axila para abducir unos grados el brazo respecto del tronco. Se trazó una línea desde el acromion hasta el olécranon, que marcó la incisión; se disecó entre el borde inferior del deltoides atrofiado y la mitad del brazo; se expusieron los músculos deltoides y tríceps; se disecó el espacio cuadrilátero, identificando por encima del músculo redondo mayor el nervio axilar y sus ramos anterior y posterior. Se continuó con la disección entre los vientres largo y lateral del tríceps hasta identificar el nervio radial y sus ramos que van a dichas porciones, bajo estimulación motora directa. Se seccionaron el nervio axilar, lo más proximalmente que fue posible y el nervio a la porción larga del tríceps, lo más distalmente que se pudo (Figura 1), con el fin de lograr una coaptación de los nervios sin tensión (Figura 2). La sutura se efectuó con dos o tres puntos de Nylon 9.0 y adhesivo de fibrina alrededor. Después de la cirugía, se utilizó inmovilizador de hombro, iniciando ejercicios de péndulo a partir de los 10 a 14 días.

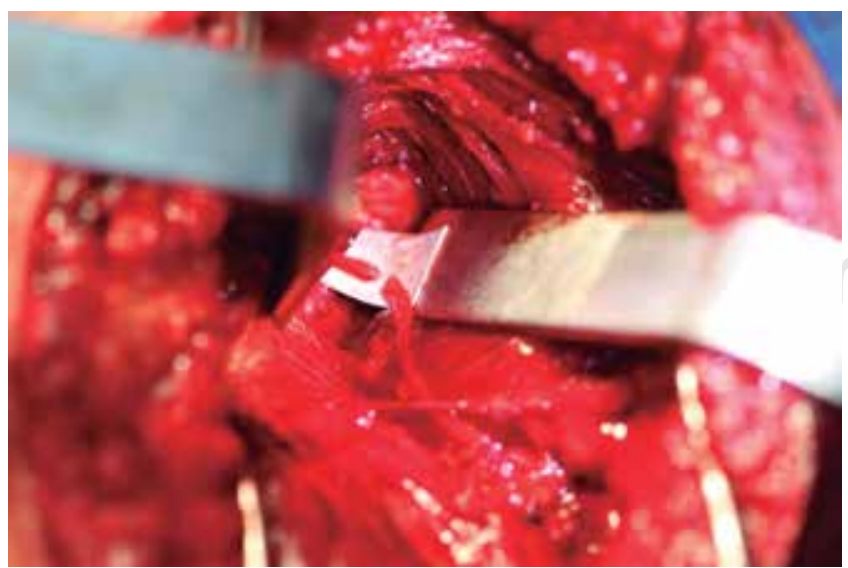

Figura 1: Visión directa de la sección realizada del nervio axilar, lo más proximalmente que fue posible y el nervio radial en la porción larga del tríceps lo más distalmente posible.
La terapia física formal se inició en promedio cuatro semanas después de la cirugía.

Se determinó en cada paciente el ángulo máximo de abducción del hombro antes de la cirugía y, posteriormente a la misma, se relevó el mismo dato de forma periódica hasta completar un mínimo de 12 meses de seguimiento. Se evaluó la eficacia del tratamiento mediante la British Medical Research Council (MRC) y el cuestionario DASH.

Se contó con la autorización del Comité Local de Investigación y Ética en Salud 3401 con número de registro R2013-3401-78.

\section{Resultados}

Un total de siete pacientes cumplieron con los criterios del estudio. En la Tabla 1 se muestran las características demográficas de la población.

La Tabla 2 presenta el mecanismo de lesión y el nivel afectado de cada paciente.

En la Tabla 3 se muestra el análisis de efectividad del tratamiento quirúrgico de la población estudiada. De estos siete pacientes, cinco se recuperaron a un nivel de resistencia M4. Hubo un resultado de cambio estadísticamente significativo entre el grado MRC preoperatoria y postoperatoria. En el análisis bivariado encontramos diferencias estadísticamente significativas con respecto al grado de mejoría de fuerza de rotación externa y de abducción medido por MRC preoperatoria y postoperatoria $(\mathrm{p}<0.05)$. Los resultados también demostraron una significancia estadística en el rango de movimiento de abducción postoperatorio de hombro $(\mathrm{p}=0.01)$. El cuestionario DASH demostró un porcentaje promedio de discapacidad de $20.29 \%$ con una funcionalidad aceptable después de 12 meses de seguimiento. Las Figuras 3 y 4 ilustran el rango de abducción preoperatoria y a los 12 meses de seguimiento del caso número 2 de la serie.

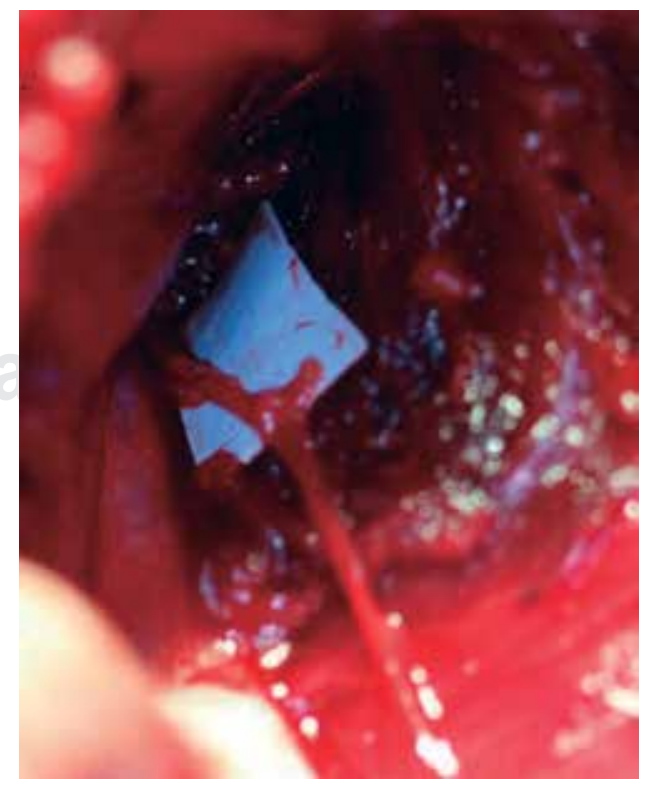

Figura 2:

Visión directa de la sutura realizada entre el nervio axilar y el nervio radial mediante sutura nylon 9.0 y adhesivo de fibrina alrededor. 


\section{Discusión}

El mecanismo de lesión de nervio axilar más habitual es el trauma cerrado que conduce a una tracción lesiva del nervio por hiperabducción, especialmente si se asocia con rotación interna del miembro superior a nivel de la articulación glenohumeral. ${ }^{1}$ La porción del nervio más vulnerable a este mecanismo es la proximal al espacio

Tabla 1: Características demográficas de la población estudiada.

$\begin{array}{lc}\text { Casos }(\mathrm{n}=7) & \mathrm{n}(\%) \\ \text { Sexo } & \\ \quad \text { Masculino } & 6(85.7) \\ \text { Femenino } & 1(14.3) \\ \text { Edad } & 51.57( \pm 14) \\ \text { Estado Civil } & 5(71.4) \\ \quad \text { Casado } & 2(28.6) \\ \text { Soltero } & \\ \text { Escolaridad } & 1(14.3) \\ \quad \text { Primaria } & 4(57.1) \\ \text { Secundaria } & 2(28.6) \\ \text { Licenciatura } & 3(42.9) \\ \text { Situación laboral } & 1(14.3) \\ \text { Activo } & 3(42.9) \\ \text { Inactivo } & \\ \text { Pensionado o jubilado } & 6(85.7) \\ \text { Lado dominante } & 1(14.3) \\ \text { Sí } & \\ \text { No } & 6(85.7) \\ \text { Lado afectado } & 1(14.3) \\ \text { Derecho } & \\ \text { Izquierdo } & \end{array}$

cuadrangular, por encontrarse adherido a estructuras adyacentes. ${ }^{1,3,11,16} \mathrm{La}$ asociación con lesiones osteoarticulares a nivel escapulohumeral y/o del manguito de los rotadores es frecuente y la incidencia varía entre $21 \%$ de la serie de 14 pacientes de Rochwerger y colaboradores y $100 \%$ de la serie de 25 pacientes de Degeorges y asociados. ${ }^{3}$ Dentro de este espectro lesivo, la luxación anteromedial del hombro parece ser la que más se asocia con la lesión del nervio axilar. ${ }^{5,13}$ En nuestro estudio se encontró que la mayoría de los casos fueron del sexo masculino en $85.7 \%$, con un promedio de edad de la población de 51.57 años, la lesión fue en la extremidad dominante en $85.7 \%$ de los casos y el mecanismo de lesión del nervio axilar más frecuente fue la luxación anteromedial en $100 \%$ secundario a una caída de su propia altura en $85.7 \%$ de los casos, lo cual es similar a lo reportado en estudios previamente publicados. ${ }^{8}$

La evaluación del daño del nervio axilar puede resultar difícil, debido a los diferentes grupos musculares que intervienen en la movilidad del hombro y a que es posible que la lesión de nervio axilar no comprometa en su totalidad la abducción del hombro. Autores como Kline ${ }^{7}$ sugieren una disminución en el rango de abducción, mientras que otros, como Bonnard ${ }^{11}$ y Sunderland, ${ }^{5}$ afirman que el compromiso es más evidente en la fuerza que en el grado de abducción. También existe discrepancia entre los autores a la hora de decidir la mejor forma de evaluar la función aislada del nervio axilar: algunos son partidarios de explorar la abducción dependiente de la porción posterior del deltoides ${ }^{12}$ y otros de explorar la extensión

\begin{tabular}{|cccc|}
\hline & \multicolumn{2}{c|}{ Tabla 2: Mecanismo y nivel de lesión de cada paciente. } \\
\hline Caso & Mecanismo & Nivel afectado & Electromiografía \\
\hline 1 & Caída/luxación hombro & Nervio axilar & Axonotmesis sin reinervación de nervio axilar \\
2 & Caída/luxación hombro & Nervio axilar & Neuropatía de fibras motoras de nervio axilar \\
3 & Caída/luxación hombro & Nervio axilar & Axonotmesis de nervio axilar \\
4 & Caída/luxación hombro & Nervio axilar & Axonotmesis de nervio axilar \\
5 & Caída/luxación hombro & Nervio axilar & Axonotmesis sin reinervación de nervio axilar \\
6 & Caída/luxación hombro & Nervio axilar & Axonotmesis sin reinervación de nervio axilar \\
7 & Colisión motocicleta/luxación hombro & Nervio axilar & Axonotmesis sin reinervación de nervio axilar \\
\hline
\end{tabular}

Tabla 3: Análisis de efectividad del tratamiento quirúrgico de pacientes con lesión de nervio axilar mediante transferencia nerviosa de rama motora radial de la cabeza medial del tríceps.

\begin{tabular}{|c|c|c|c|c|c|c|c|c|c|}
\hline Caso & Sexo/Edad & Lado & $\begin{array}{l}\text { Intervalo } \\
\text { entre lesión y } \\
\text { tratamiento }\end{array}$ & $\begin{array}{l}\text { Fuerza deltoidea } \\
\text { y de rotación } \\
\text { externa } \\
\text { preoperatoria }\end{array}$ & $\begin{array}{l}\text { Fuerza deltoidea y } \\
\text { de rotación externa } \\
\text { tras } 12 \text { meses de } \\
\text { seguimiento }\end{array}$ & $\begin{array}{l}\text { Abducción } \\
\text { preoperatoria } \\
\text { máxima }\end{array}$ & $\begin{array}{l}\text { Abducción } \\
\text { postoperatoria } \\
\text { tras } 12 \text { meses de } \\
\text { seguimiento }\end{array}$ & Variación & $\begin{array}{c}\text { Cuestionario } \\
\text { DASH }\end{array}$ \\
\hline 1 & $\mathrm{M} / 52$ & $\mathrm{D}$ & 6 Meses & M0 & M3 & $20^{\circ}$ & $45^{\circ}$ & $+25^{\circ}$ & $22.05 \%$ \\
\hline 2 & $\mathrm{M} / 53$ & I & 11 Meses & M0 & M4 & $80^{\circ}$ & $100^{\circ}$ & $+20^{\circ}$ & $10.29 \%$ \\
\hline 3 & $\mathrm{M} / 44$ & $\mathrm{D}$ & 17 Meses & M0 & M4 & $70^{\circ}$ & $85^{\circ}$ & $+15^{\circ}$ & $11.02 \%$ \\
\hline 4 & $\mathrm{~F} / 49$ & $\mathrm{D}$ & 6 Meses & M0 & M4 & $30^{\circ}$ & $90^{\circ}$ & $+60^{\circ}$ & $20.00 \%$ \\
\hline 5 & $\mathrm{M} / 63$ & $\mathrm{D}$ & 8 Meses & M0 & M3 & $45^{\circ}$ & $80^{\circ}$ & $+35^{\circ}$ & $25.83 \%$ \\
\hline 6 & $\mathrm{M} / 73$ & $\mathrm{D}$ & 13 Meses & M0 & M4 & $60^{\circ}$ & $90^{\circ}$ & $+30^{\circ}$ & $30.83 \%$ \\
\hline 7 & $\mathrm{M} / 27$ & $\mathrm{D}$ & 9 Meses & M0 & M4 & $45^{\circ}$ & $50^{\circ}$ & $+5^{\circ}$ & $22.05 \%$ \\
\hline
\end{tabular}




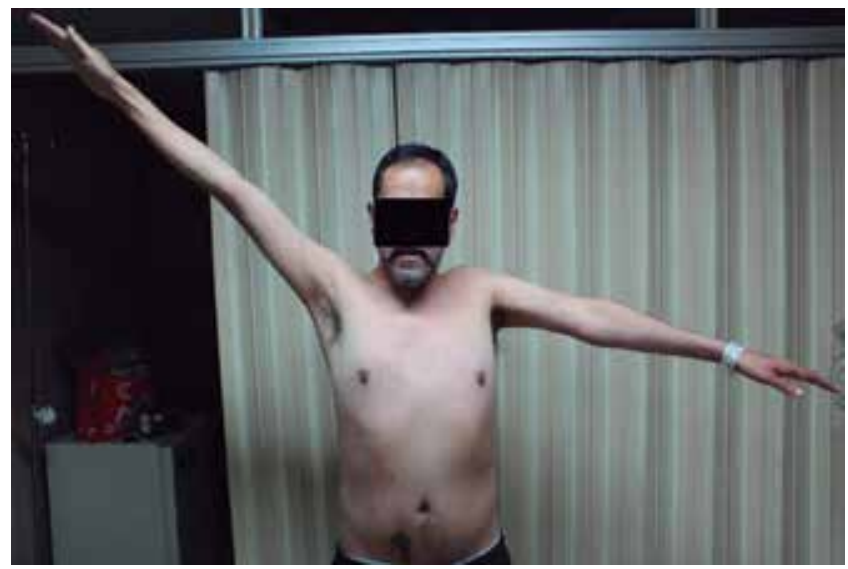

Figura 3: Fotografía que muestra la abducción del hombro izquierdo preoperatoria del caso número 2.

completa del brazo..$^{20}$ Ante una pérdida completa de abducción se debe sospechar una lesión asociada del nervio supraescapular y/o del manguito de los rotadores. ${ }^{16}$ Se estima aproximadamente en $62 \%$ la incidencia de lesión nerviosa en los estudios electrofisiológicos tras un traumatismo en el hombro y el nervio axilar es el que se afecta con más frecuencia. ${ }^{14}$ En nuestro estudio se encontró que siete de los casos a su ingreso mostraron una fuerza deltoidea y de rotación externa preoperatoria MO del Medical Research Council, una disminución en el rango de movilidad de hombro en abducción con un promedio de movilidad de 50 grados y un estudio de electromiografía con un resultado positivo para lesión de nervio axilar en $100 \%$ de los casos, lo cual es similar a los hallazgos clínicos y electromiográficos reportados en series de casos previas. ${ }^{11}$

La mayoría de estas lesiones evolucionan espontáneamente hacia la recuperación en los primeros seis meses y la parálisis persiste en menos de $20 \%$ de los casos. ${ }^{4,5}$ Cuando la parálisis del nervio axilar persiste clínicamente sin evidencia de recuperación neurofisiológica, se recomienda el tratamiento quirúrgico antes de que transcurran seis meses. ${ }^{14}$ La reparación del nervio y su recuperación funcional evitan a largo plazo el desarrollo de una lesión secundaria a nivel del manguito rotador, la degeneración de la articulación escapulohumeral minimiza el impacto estético de la atrofia del músculo deltoides. ${ }^{19}$ La población de nuestro estudio fueron intervenidos quirúrgicamente en un rango de tiempo de entre 6 y 17 meses, con un promedio de tiempo para el tratamiento de 10 meses. Esto demuestra que en nuestro país el tratamiento para las lesiones de nervio axilar tiene un tratamiento quirúrgico tardío, lo que favorece el desarrollo de lesiones de mango rotador, degeneración de la articulación escapulohumeral ${ }^{3}$ y atrofia del músculo deltoides, lo que demuestra la discrepancia con lo recomendado por las series de casos previamente publicadas, lo cual puede ser debido a que los pacientes son derivados de manera tardía a nuestra unidad para manejo definitivo al no contar con cirujano experto en tratar lesiones de nervio axilar.

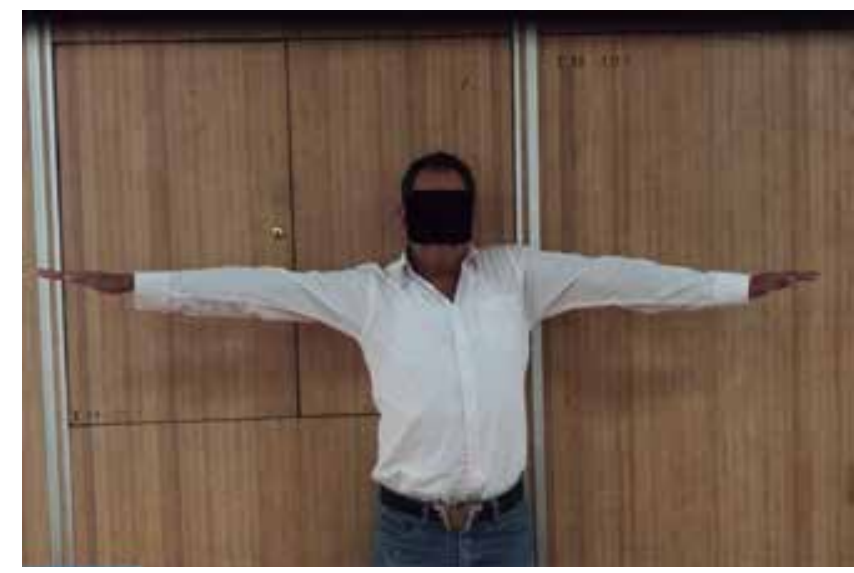

Figura 4: Fotografía que muestra el resultado postoperatorio de hombro izquierdo del caso número 2 .

Del total de nuestros casos, cinco se recuperaron a un nivel de resistencia M4. En el análisis bivariado encontramos diferencias estadísticamente significativas con respecto al grado de mejoría de fuerza de rotación externa y de abducción medido por MRC preoperatoria y postoperatoria $(\mathrm{p}<0.05)$. Los resultados también demostraron una significancia estadística en el rango de movimiento de abducción postoperatorio de hombro $(\mathrm{p}=0.01)$. En estudios realizados previamente por Leechavengvongs y colaboradores se encontró un grado de mejoría de abducción de 115 grados en promedio, lo cual contrasta con lo encontrado en nuestro trabajo, que demuestra un porcentaje promedio de mejoría de 27 grados a los 12 meses de seguimiento.

El cuestionario DASH demostró un porcentaje promedio de discapacidad de $20.29 \%$ después de 12 meses de seguimiento, lo cual nos da a conocer que, a pesar del tratamiento quirúrgico tardío, la funcionalidad de nuestros pacientes es buena, por lo que pueden regresar a realizar sus actividades con normalidad.

Este estudio tiene varias limitaciones. Su naturaleza retrospectiva debilitó inherentemente el poder de análisis. Un mínimo de 12 meses de seguimiento puede no ser suficiente para determinar la recuperación final a largo plazo. A pesar de su amplia aceptación, pruebas manuales de fuerza muscular, MRC y cuestionario DASH pueden afectar la calificación al ser medidas subjetivamente por los evaluadores. No obstante estas limitaciones, nuestro estudio demostró que la lesión aislada del nervio axilar puede ser tratada con éxito mediante transferencia nerviosa de rama motora radial de la cabeza medial del tríceps.

Los puntos fuertes de nuestro estudio son el seguimiento consistente de todos los casos estudiados después del tratamiento quirúrgico con una transferencia nerviosa radial a nervio axilar, el registro sistemático de todas las variables médicas y quirúrgicas que pueden estar asociadas con la eficacia del tratamiento quirúrgico, además de la evaluación con el cuestionario DASH que permite demostrar qué tan funcional es un paciente después de su reincorporación a 
sus actividades de la vida diaria. Se justifica la realización de nuevos estudios prospectivos con tamaños de muestra más amplios y un mayor poder estadístico. Esta información podría ayudarnos a proporcionar un mejor asesoramiento a nuestros pacientes con respecto al pronóstico y eventualmente redefinir nuestras estrategias de tratamiento para el manejo de las lesiones traumáticas aisladas de nervio axilar.

\section{Conclusión}

Nuestro estudio retrospectivo encontró que las lesiones traumáticas aisladas del nervio axilar son infrecuentes en nuestra unidad. Que la técnica más empleada para su manejo es la neurotización con el nervio radial como donador, consideramos que es una técnica sencilla y demostró mejores resultados que técnicas clásicas descritas en la literatura. Se demostró que el tratamiento quirúrgico en pacientes que no muestran signos de reinervación neurofisiológica del nervio axilar transcurridos seis meses desde el traumatismo tienen un manejo tardío en nuestra unidad.

\section{Bibliografía}

1. Domínguez-Pérez M, Socolovsky M, Di Masi G, Arráez-Sánchez MA. Lesiones traumáticas aisladas del nervio axilar. Experiencia en 4 casos de transferencia nerviosa radial y revisión de la literatura. Neurocirugía [Internet]. 2012; 23(6): 226-33. Disponible en: http:// dx.doi.org/10.1016/j.neucir.2012.06.003

2. Petrucci FS, Morelli A, Raimondi PL. Axillary nerve injuries--21 cases treated by nerve graft and neurolysis. J Hand Surg Am [Internet]. 1982; 7(3): 271-8. Available from: http://dx.doi.org/10.1016/S03635023(82)80178-0

3. Desai MJ, Daly CA, Seiler JG, Wray WH, Ruch DS, Leversedge FJ. Radial to axillary nerve transfers: a combined case series. J Hand Surg Am [Internet]. 2016; 41(12): 1128-34. Available from: http://dx.doi. org/10.1016/j.jhsa.2016.08.022

4. Addas BM, Midha R. Nerve transfers for severe nerve injury. Neurosurg Clin N Am [Internet]. 2009; 20(1): 27-38. Available from: http://dx.doi.org/10.1016/j.nec.2008.07.018

5. Leechavengvongs $\mathrm{S}$, Witoonchart K, Uerpairojkit $\mathrm{C}$, Thuvasethakul $\mathrm{P}$. Nerve transfer to deltoid muscle using the nerve to the long head of the triceps, part II: a report of 7 cases. J Hand Surg Am. 2003; 28(4): 633-8.
6. Alnot JY, Valenti P. Surgical repair of the axillary nerve. Apropos of 37 cases. Int Orthop. 1991; 15(1): 7-11.

7. Oberlin C, Durand S, Belheyar Z, Shafi M, David E, Asfazadourian H. Nerve transfers in brachial plexus palsies. Chir Main. 2009; 28(1): 1-9.

8. Tung TH, Mackinnon SE. Nerve transfers: indications, techniques, and outcomes. J Hand Surg Am [Internet]. 2010; 35(2): 332-41. Available from: http://dx.doi.org/10.1016/j.jhsa.2009.12.002

9. Leechavengvongs S, Witoonchart K, Uerpairojkit C, Thuvasethakul P, Malungpaishrope K. Combined nerve transfers for $\mathrm{C} 5$ and $\mathrm{C} 6$ brachial plexus avulsion injury. J Hand Surg Am. 2006; 31(2): 183-9.

10. Bertelli JA, Ghizoni MF. Transfer of the accessory nerve to the suprascapular nerve in brachial plexus reconstruction. J Hand Surg Am. 2007; 32(7): 989-98.

11. Leechavengvongs S, Malungpaishorpe K, Uerpairojkit C, Ng CY, Witoonchart K. Nerve transfers to restore shoulder function. Hand Clin [Internet]. 2016; 32(2): 153-64. Available from: http://dx.doi. org/10.1016/j.hcl.2015.12.004

12. Miller JH, Garber ST, McCormick DE, Eskandari R, Walker ML, Rizk E, et al. Oberlin transfer and partial radial to axillary nerve neurotization to repair an explosive traumatic injury to the brachial plexus in a child: case report. Childs Nerv Syst. 2013; 29(11): 2105-9.

13. Bertelli JA, Santos MA, Kechele PR, Ghizoni MF, Duarte H. Triceps motor nerve branches as a donor or receiver in nerve transfers. Neurosurgery. 2007; 61(5 Suppl 2): 333-8; discussion 338-9.

14. Wood MB, Murray PM. Heterotopic nerve transfers: recent trends with expanding indication. J Hand Surg Am. 2007; 32(3): 397-408.

15. Uz A, Apaydin N, Bozkurt M, Elhan A. The anatomic branch pattern of the axillary nerve. J Shoulder Elbow Surg. 2007; 16(2): 240-4.

16. Galvin JW, Eichinger JK. Outcomes following closed axillary nerve injury: a case report and review of the literature. Mil Med [Internet]. 2016; 181(3): e291-7. Available from: http://publications.amsus.org/ doi/10.7205/MILMED-D-15-00205

17. Witoonchart K, Leechavengvongs S, Uerpairojkit C, Thuvasethakul $\mathrm{P}$, Wongnopsuwan $\mathrm{V}$. Nerve transfer to deltoid muscle using the nerve to the long head of the triceps, part I: an anatomic feasibility study. $J$ Hand Surg Am. 2003; 28(4): 628-32.

18. Erhardt AJ, Futterman B. Variations in the innervation of the long head of the triceps brachii: a cadaveric investigation. Clin Orthop Relat Res. 2017; 475(1): 247-50.

19. Hervás MT, Navarro-Collado MJ, Peiró S, Pérez JLR, López-Matéu $\mathrm{P}$, Martínez-Tello I. Versión española del cuestionario DASH. Adaptación transcultural, fiabilidad, validez y sensibilidad a los cambios. Med Clin (Barc) [Internet]. 2006; 127(12): 441-7. Disponible en: http://linkinghub.elsevier.com/retrieve/pii/S0025775306722898

20. Craig P, Dieppe P, Macintyre S, Michie S, Nazareth I, Petticrew M. Developing and evaluating complex interventions: the new Medical Research Council guidance. BMJ [Internet]. 2008; 337: a1655. Available from: http://www.bmj.com/lookup/doi/10.1136/bmj.a1655 